\title{
Prevalence of left ventricular systolic dysfunction by single echocardiographic view: towards an evidence-based point of care cardiac ultrasound scanning protocol
}

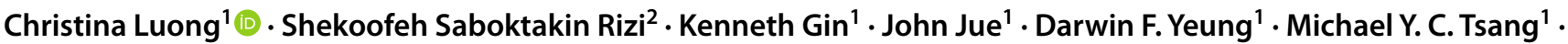 \\ Eric C. Sayre ${ }^{3} \cdot$ Teresa S. M. Tsang $^{1}$
}

Received: 20 July 2021 / Accepted: 26 October 2021 / Published online: 2 November 2021

(c) Crown 2021

\begin{abstract}
Limited views are often obtained in the setting of cardiac ultrasound, however, the likelihood of missing left ventricular (LV) dysfunction based on a single view is not known. We sought to determine the echo views that were least likely to miss LV systolic dysfunction in consecutive transthoracic echocardiograms (TTEs). Structured data from TTEs performed at 2 hospitals from September 25, 2017, to January 15, 2019, were screened. Studies of interest were those with reported LV dysfunction. Views evaluated were the parasternal long-axis (PLAX), parasternal-short axis at mitral (PSAX M), papillary muscle (PSAX PM), and apical (PSAX A) levels, apical 2 (AP2), apical 3 (AP3), and apical 4 (AP4) chamber views. The probability that a view contained at least 1 abnormal segment was determined and analyzed with McNemar's test for 21 adjusted pair-wise comparisons. There were 4102 TTE studies included for analysis. TTEs on males comprised $72.7 \%$ of studies with a mean LV ejection fraction of $42.8 \pm 9.7 \%$. The echo view with the greatest likelihood of encompassing an abnormal segment was the AP2 view with a prevalence of $93.4 \%$ ( $<<0.001$, compared to all other views). The PLAX view performed the worst with a prevalence of $82.5 \%(\mathrm{p}<0.015$, compared to all other views). The best parasternal view for the detection of abnormality was the PSAX PM view at $90.4 \%$. In conclusions, a single echo view will contain abnormal segments $>82 \%$ of the time in the setting of LV systolic dysfunction, with a prevalence of up to $93.4 \%$ in the apical windows.
\end{abstract}

Keywords Echocardiography $\cdot$ Left ventricular dysfunction $\cdot$ Regional wall motion abnormality $\cdot$ Cardiac ultrasound

$\begin{array}{ll}\text { Abbreviations } \\ \text { AP2 } & \text { Apical } 2 \text { chamber } \\ \text { AP3 } & \text { Apical } 3 \text { chamber } \\ \text { AP4 } & \text { Apical } 4 \text { chamber } \\ \text { LVEF } & \text { Left ventricular ejection fraction } \\ \text { TTE } & \text { Transthoracic echocardiogram } \\ \text { PLAX } & \text { Parasternal long-axis } \\ \text { POCUS } & \text { Point of care ultrasound } \\ \text { PSAX A } & \begin{array}{l}\text { Parasternal short-axis view at the level of the } \\ \text { apex }\end{array}\end{array}$

Christina Luong

christina.luong@ubc.ca

1 Division of Cardiology, University of British Columbia, Vancouver, BC, Canada

2 Department of Medicine, University of British Columbia, Vancouver, BC, Canada

3 Arthritis Research Canada, Richmond, BC, Canada

\begin{abstract}
PSAX M Parasternal short-axis view at the level of the mitral valve

PSAX PM Parasternal short-axis view at the level of the papillary muscle

RWMA Regional wall motion abnormality
\end{abstract}

\section{Introduction}

Cardiac ultrasound is a widely available and convenient imaging modality that has become a standard component of medical school and residency training [1-6]. The purpose of cardiac ultrasound at the bedside is to allow for the rapid assessment of cardiac structure and function in timesensitive settings. Given the brisk uptake of cardiac point of care ultrasound (POCUS), abbreviated protocols have been adopted to ensure that this supplement to the physical exam can be completed quickly as a part of routine care. The 
clinical utility of cardiac POCUS has been demonstrated in numerous investigations [7-14] and is a tool that has been especially relevant in the setting of the COVID-19 pandemic $[15,16]$. Various guidelines have been published by different specialty groups including critical care, emergency medicine, and cardiology for the training and targets of cardiac ultrasound [17-21].

Though not uniformly accepted, the evaluation of regional wall motion abnormalities (RWMA) has been included as a target of cardiac POCUS in critical care [18, 21]. Identification of LV dysfunction and RWMA is of significant interest to numerous specialties and may alter care pathways and triaging. The likelihood that abnormal segments will be visualized using only limited cardiac views is not known. We sought to evaluate the proportion of time that a single standard echocardiographic view would contain abnormal LV segments when LV dysfunction was present to determine the view that would be least likely to miss abnormalities. These results may have implications for training, leading to the practice of imaging views of highest yield. Additionally, clinician scanners will have a greater awareness of the probability of missing LV dysfunction in the setting of limited cardiac ultrasound images.

\section{Material and methods}

Procedures and protocols were approved by the institutional review board for all aspects of the study. A waiver of consent was approved for this study.

\section{Transthoracic echocardiogram case selection}

The transthoracic echos (TTE) of interest were studies completed at Vancouver General Hospital and University of British Columbia Hospital from September 25, 2017, to January 15,2019 . Transesophageal and stress echocardiograms were not included for the purposes of this analysis. Studies with any of the 16 segments scored as 0 , unable to grade due to quality or technical factors, were excluded. Structured data was obtained from the reporting database and no images were over-read for the purpose of this study.

\section{Echocardiographic examinations}

All TTEs were performed by professional sonographers on commercially available echocardiographic platforms using phased-array transducers. The TTE studies were acquired and interpreted in accordance with the American Society of echocardiography standards [22] and ultrasound enhancement agent was utilized as clinically indicated. Structured data used for analysis in the study were extracted from the clinical record, reported by experienced echocardiographers with level 3 training (6 interpreters). Each of the $16 \mathrm{LV}$ wall segments was coded from 1 to 5 based on the following criteria [22]: Normal $=1$ (systolic increase in thickening greater than $40 \%$ ), hypokinesis $=2$ (less than $40 \%$ thickening), severe hypokinesis or akinesis $=3$ (less than $10 \%$ thickening), dyskinesis (paradoxical systolic motion away from the center of the $\mathrm{LV}$ ) $=4$, and aneurysmal (diastolic deformation $)=5$. These standard segmental labels were documented in the echocardiography database as part of the clinical report.

\section{Data review and imputations}

Following the export of structured TTE data, the entries were reviewed for comprehensiveness. In TTEs where none of the 16 segments were labeled, all segments were imputed as (1) normal if the LVEF was $>52 \%$ and (2) hypokinetic if the LVEF was $<52 \%$. The threshold of LVEF $>52 \%$ was considered normal based on current guidelines from the American Society of Echocardiography [22]. Studies with the absence of labelled RWMA and LVEF greater than 52\% were considered normal. Analysis for the probability of a view containing abnormal segments was only performed on TTEs regarded as abnormal: $\mathrm{LVEF}<52 \%$ or the presence of at least 1 segment of RWMA.

\section{Calculation of single-view prevalence for abnormal segments}

The echo views of interest for this study were obtained from parasternal and apical windows. These views are key components of a standard TTE. The 7 views chosen were the parasternal long-axis (PLAX), parasternal short axis at the mitral valve level (PSAX M), parasternal short axis at the papillary muscle level (PSAX PM), parasternal short axis at the apex (PSAX A), apical 2 chamber (AP2), apical 3 chamber (AP3), and apical 4 chamber (AP4) view. A schematic of the component segments of each view is shown in Fig. 1. Only TTEs with at least 1 segment that was designated as abnormal (segment score greater than 1) were used in the evaluation of single-view probability for detection of LV dysfunction. For each of the 7 TTE views, we calculated the proportion of time that a view would contain segments that would demonstrate abnormalities, providing the marginal percentages for the purpose of analysis.

\section{Statistical analysis}

McNemar's test was adjusted for 21 pair-wise comparisons against the 7 views for the likelihood of detecting LV dysfunction. All analyses were performed overall and separated into males and females for subgroup analysis. Comparison of differences in proportions based on sex was evaluated 


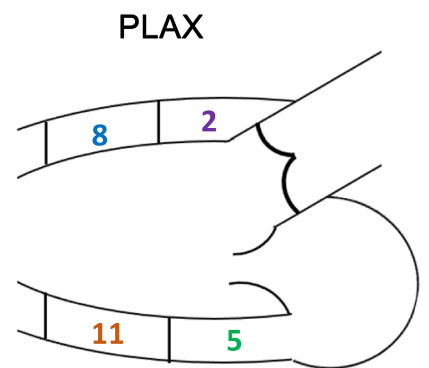

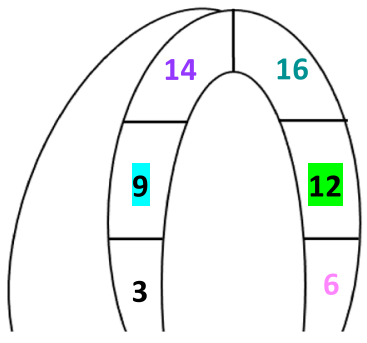

AP4
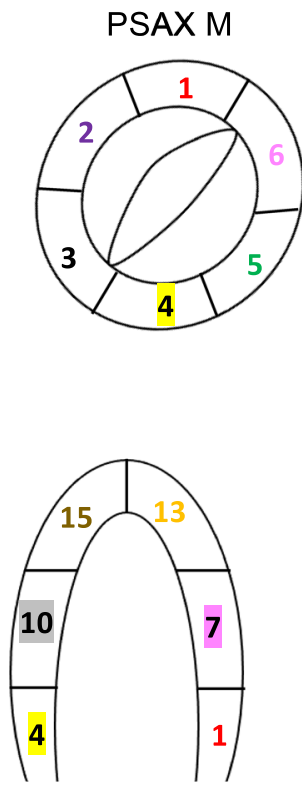

AP2
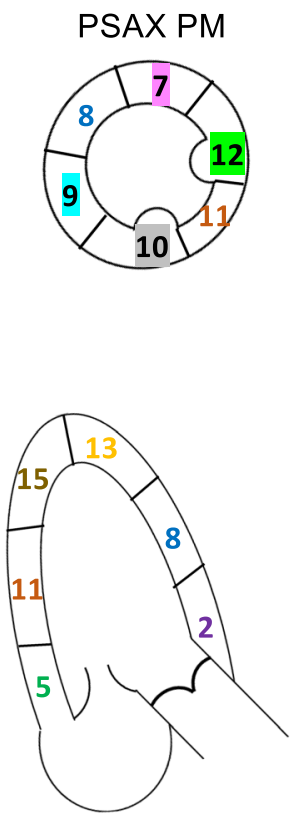

AP3
PSAXA

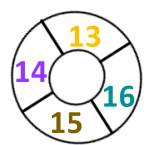

1 Basal anterior

2 Basal anteroseptal

3 Basal inferoseptal

4 Basal inferior

5 Basal inferolateral

6 Basal anterolateral

7 Mid anterior

8 Mid anteroseptal

9 Mid inferoseptal

10 Mid inferior

11 Mid inferolateral

$12 \mathrm{Mid}$ anterolateral

3 Apical anterior

14 Apical septal

15 Apical inferior

16 Apical lateral
Fig. 1 Echocardiographic 16 segment model. Schematic diagram of each of the 7 echo views of interest and the segments included in each view. $A P 2$ apical 2 chamber view, $A P 3$ apical 3 chamber view, $A P 4$ apical 4 chamber view, PLAX parasternal long-axis view, PSAX
$A$ parasternal short-axis view at the level of the apex; PSAX $M$ parasternal short-axis view at the level of the mitral valve, PSAX PM parasternal short-axis view at the level of the papillary muscle

the time) and the segment least likely to be coded as abnormal was the basal anterolateral segment (55.9\% of the time). A summary of the distribution of abnormalities by segment is shown in Supplemental Fig. 1 and 2.

\section{Single-view probability for containing abnormal LV segments}

There were 4102 TTEs that met inclusion criteria. Using the 7 previously described views of interest, the proportion of time that a view would contain at least 1 abnormal segment was as follows: PLAX 82.5\%, PSAX M 86.5\%, PSAX PM 90.4\%, PSAX A 84.7\%, AP2 93.4\%, AP3 90.4\%, and AP4 $90.4 \%$ (Fig. 2). Overall, the PLAX view was the least likely to include abnormal LV segments $(\mathrm{p}<0.015$, compared to all other views). The parasternal view that was most likely to demonstrate LV dysfunction was the PSAX PM which was significantly better than the PSAX A $(p<0.001)$ and PSAX $\mathrm{M}(\mathrm{p}<0.001)$ views and comparable to the AP3 $(\mathrm{p}=1.0)$ and AP4 views $(p=1.0)$. The AP2 chamber view performed best overall and was superior to the AP3 $(p<0.001)$ and AP4 $(p<0.001)$ views. Summary of study findings shown in Fig. 3. 


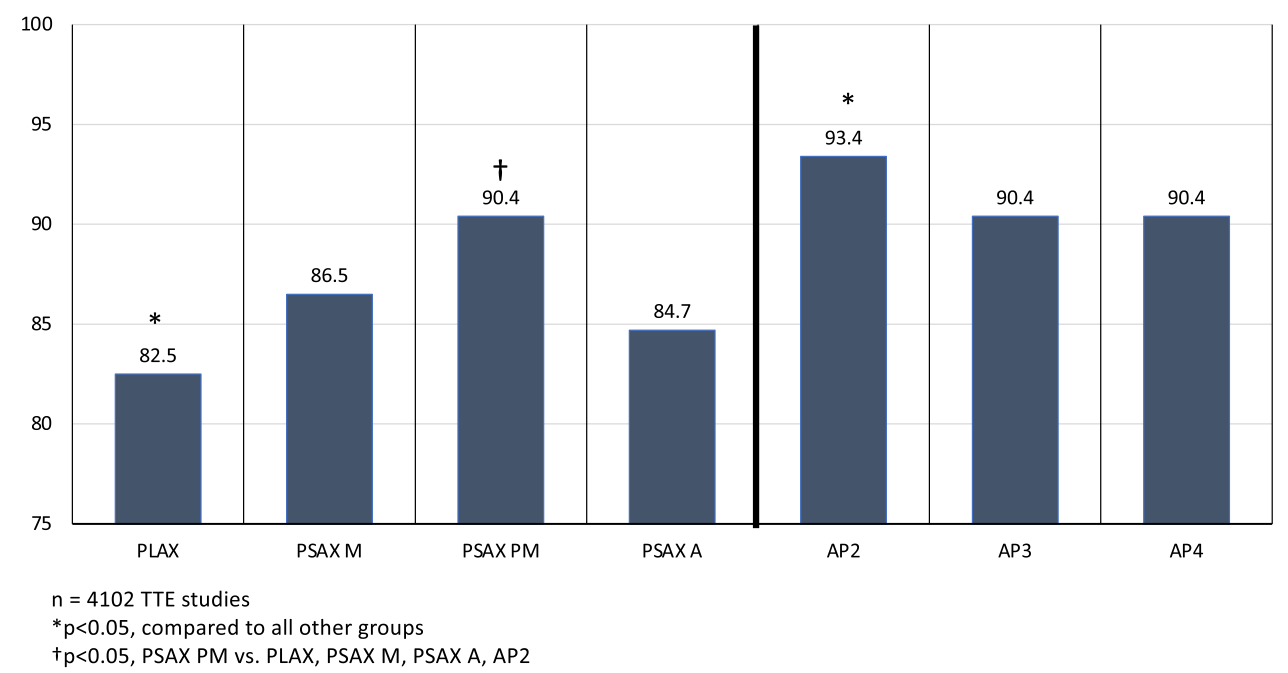

Fig. 2 Single echo view prevalence of abnormal left ventricular systolic function. Percentage of time that a single view will contain at least 1 segment coded as abnormal when left ventricular dysfunction is present. McNemar's test with 21 adjusted pair-wise comparisons. ${ }^{*} \mathrm{p}<0.05$, compared to all other groups; ${ }^{\dagger} \mathrm{p}<0.05$, PSAX PM vs. PLAX, PSAX M, PSAX A, AP2. AP2 had the greatest likelihood of including an abnormal segment and the PLAX was least likely to include an abnormal segment. $A P 2$ apical 2 chamber view, $A P 3$ apical 3 chamber view, AP4 apical 4 chamber view, PLAX parasternal long-axis view, PSAX A parasternal short-axis view at the level of the apex; PSAX M parasternal short-axis view at the level of the mitral valve, PSAX PM parasternal short-axis view at the level of the papillary muscle

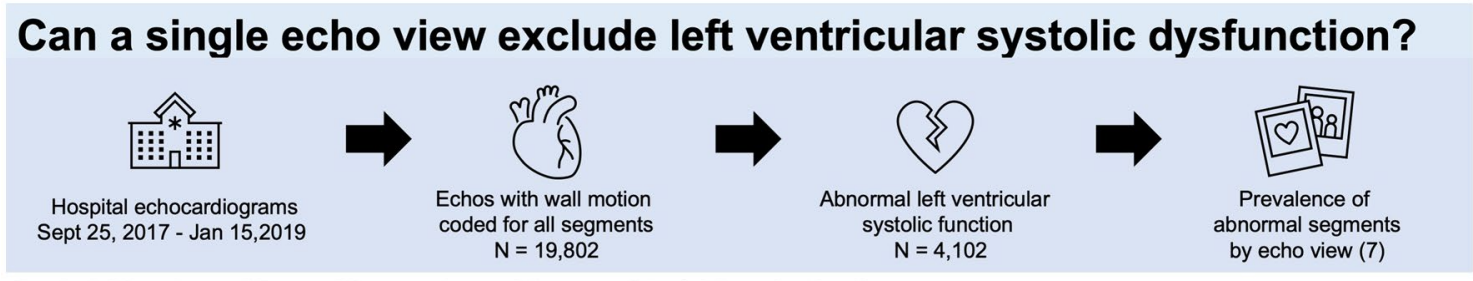

Probability that a View will contain an Abnormal Left Ventricular Segment:
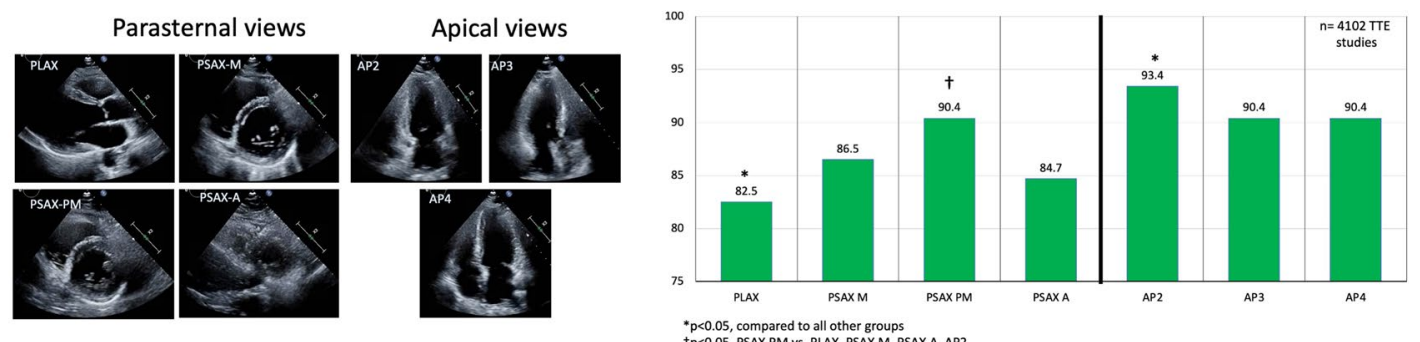

CONCLUSION:

-A single echo view will contain abnormal segments $>82 \%$ of the time when at least 1 abnormal segment is present

- The apical 2 chamber view had the greatest likelihood of including abnormal segments $(93.4 \%)$ and the parasternal long-axis view was least likely to contain abnormal segments $(82.5 \%)$ in the setting of left ventricular dysfunction

Fig. 3 Graphical summary of study findings

When these results were examined by sex, the overall result was similar but there were differences in LV abnormality prevalence for 2 views between males and females (Fig. 4). In females, the PSAX PM view was significantly less likely to include abnormalities as compared to males
$88.3 \%$ versus $91.2 \%(\mathrm{p}=0.005)$ and was not superior to the PSAX A and PSAX M views. Additionally, the AP4 view was more likely to contain LV abnormalities in females compared to males, $91.7 \%$ versus $89.8 \%(p=0.006)$, performing comparably to the AP2 view $(93.3 \%, \mathrm{p}=1)$. 


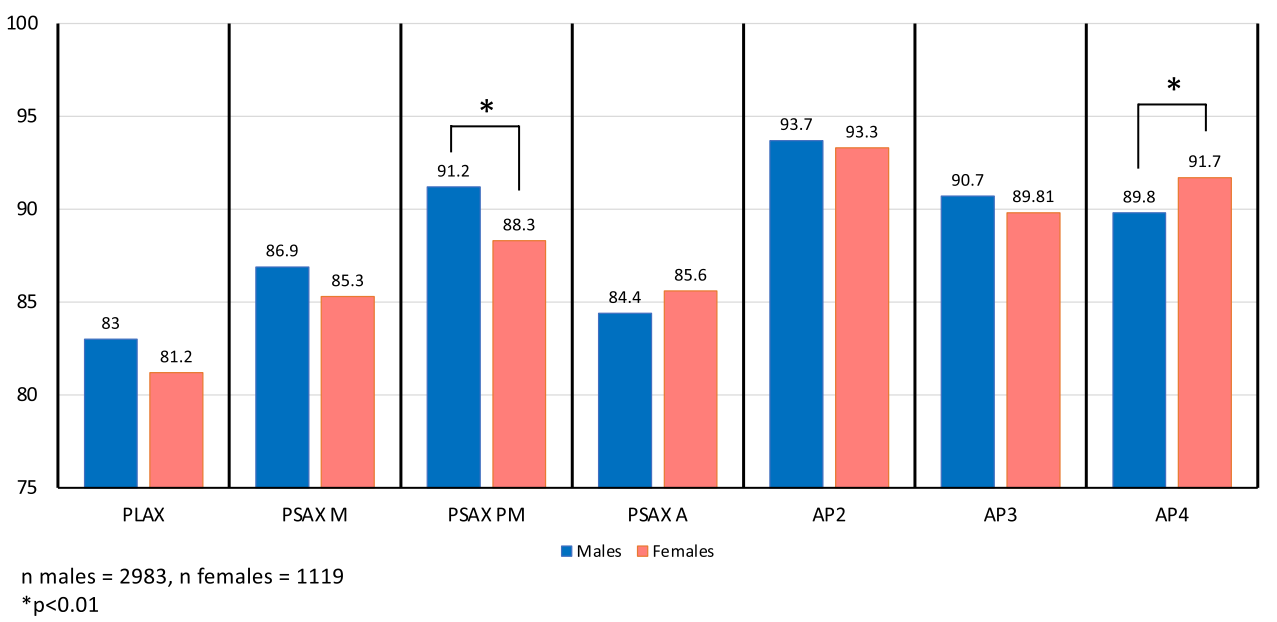

Fig. 4 Single echo view prevalence of abnormal left ventricular systolic function by sex. Percentage of time that a single view will contain at least 1 segment coded as abnormal when left ventricular dysfunction is present by sex. 2 tailed z-test was performed to determine the difference in proportion between sexes. $* p<0.01$. There was a lower prevalence of abnormal segments in females for the PSAX PM $(p=0.00496)$ and a greater prevalence of abnormal segments in females for the AP4 $(p=0.00614)$. The AP2 view demonstrated LV

\section{Single-view probability for containing abnormal LV segments: RWMA subgroup}

Similar results are observed when examining echocardiograms reporting RWMA, excluding samples designated as global dysfunction. There were 2072 TTEs with a mean age of $68.6 \pm 12.7$ years, LVEF $44.4 \pm 10.0 \%$, and $75.6 \%$ male. Using the 7 views of interest, the proportion of time that a view would contain at least 1 abnormal segment was as follows: PLAX 65.4\%, PSAX M 73.2\%, PSAX PM 80.9\%, PSAX A $69.8 \%$, AP2 87.3\%, AP3 81.0\%, and AP4 80.9\%. Again, the PLAX view was the least likely to include abnormal LV segments ( $p<0.015$, compared to all other views) and the AP2 chamber view performed best overall $(\mathrm{p}<0.001$, compared to all other views).

\section{Single-view probability for containing abnormal LV segments: LVEF $<\mathbf{4 0 \%}$ subgroup}

When focusing on a sample with abnormal LV systolic function (global or RWMA) and LVEF $<40 \%$, there are slight differences in view performance. There were 1338 TTEs with males making up $75.9 \%$ of the cohort. The proportion of time that a view would contain at least 1 abnormal segment was as follows: PLAX 96.5\%, PSAX M 94.3\%, PSAX PM 99.2\%, PSAX A 96.5\%, AP2 98.0\%, AP3 98.9\%, and AP4 $97.9 \%$. The view that was least likely to include abnormal LV segments was the PSAX M ( $p<0.001$, compared to all other views except the PSAX A view). The view with best dysfunction most frequently regardless of sex with similar probability of containing an abnormal segment. AP2 apical 2 chamber view, AP3 apical 3 chamber view, AP4 apical 4 chamber view, $P L A X$ parasternal long-axis view, PSAX A parasternal short-axis view at the level of the apex; PSAX M parasternal short-axis view at the level of the mitral valve, PSAX PM parasternal short-axis view at the level of the papillary muscle

performance was the PSAX PM view though there was no statistical difference compared with the AP2 and AP3 view. The PSAX PM view was significantly better than the PLAX $(\mathrm{p}<0.001)$, PSAX M $(\mathrm{p}<0.001)$, PSAX A $(\mathrm{p}<0.001)$, and AP4 view $(\mathrm{p}=0.028)$.

\section{Single-view probability for containing abnormal LV segments: LVEF $\geq \mathbf{4 0} \%$ subgroup}

For echocardiograms with abnormal LV systolic function (global or RWMA) and LVEF $>40 \%$ there were 2764 samples with males making up $71.2 \%$ of the cohort. The proportion of time that a view would contain at least 1 abnormal segment was as follows: PLAX 75.7\%, PSAX M 82.7\%, PSAX PM 86.1\%, PSAX A 79.1\%, AP2 91.4\%, AP3 86.3\%, and AP4 $86.8 \%$. The view that was least likely to include abnormal LV segments was the PLAX view ( $\mathrm{p}<0.008$, compared to all other views). The view with the best performance was the $\operatorname{AP} 2(p<0.001$, compared to all other views).

\section{Combination view probability for detection of abnormal LV segments}

Recognizing that LV systolic dysfunction is best detected with multiple views, additional analysis was performed to determine the second view that would be of highest yield. The AP2 view failed to detect $L V$ dysfunction in $6.6 \%$ of samples when abnormalities were present. The view that was most likely to detect the remaining abnormalities was the 
AP4 view, picking up an additional $78.4 \%$. When the AP2 and AP4 view are used in combination, $98.6 \%$ of samples with LV systolic dysfunction were detected in this cohort. As an alternate to the apical windows, the best parasternal window for a second view would be the PSAX PM which would detect an additional $76.5 \%$ of missed samples. When used in combination, the AP2 and PSAX PM view would detect $98.5 \%$ of samples with LV systolic dysfunction.

\section{Discussion}

The principal findings of the study were: (1) in patients with LV systolic dysfunction, a single echocardiographic view would include abnormal LV segments over $82 \%$ of the time, (2) the best parasternal short-axis window is at the papillary muscle level (PSAX-PM), and (3) the apical views are more likely to include LV abnormalities when one is present. This study demonstrates that even single views obtained on echo can be useful for the evaluation of LV systolic abnormalities with a relatively low probability of excluding abnormal segments. However, given the lower likelihood of including abnormal segments using the PLAX view, acquisition from the apical windows would be advised if only limited images can be acquired due to technical or clinical factors. Given the lower number of segments included in the PLAX and PSAX A views, it is not unexpected that these would be the views that are of the lowest yield, however, the distribution of RWMA is not uniform. Furthermore, the etiology for RWMA is mostly likely secondary to coronary artery disease which is more likely to impact the distal segments. Therefore, the apical views have been expected to be of greatest likelihood of including abnormalities due to the presence of the apical segments. Our finding highlights the need for proficiency in apical window scanning even in clinician scanners who would typically acquire only limited images. These results quantify the probability of missing LV systolic dysfunction if relying on single views and supports the standard practice of attempting to acquire multiple views to improve accuracy and diagnostic confidence.

This study is technically theoretical in that it examines the prevalence of RWMA that would be expected to be visualized in each respective view. The labels for abnormalities themselves are derived from full TTE studies, typically with over 100 video loops that allowed for comprehensive evaluation of each LV myocardial segment. We chose to focus on only abnormal studies to determine the likelihood of missing abnormalities using a single echo view. The abnormality prevalence we report would be dependent on adequate image quality for each view and visualization of every segment which may not have been possible in practice. The values calculated in our study is a reflection of the real-world distribution of RWMAs in consecutive patients at a tertiary care hospital. The prevalence and territories of RWMA will depend on the population and the indication for echocardiogram as demonstrated in other investigations for prediction of acute coronary syndrome. In a cohort without prior revascularization admitted with non-ST elevation myocardial infarction, $52 \%$ of patients had RWMA on echocardiogram with the most commonly impacted segments in the inferior distribution (31.7\%) [23]. Similar results were observed in a study with prehospital echo performed by emergency physicians. In patients with confirmed subsequent NSTEMI, the most frequent RWMA localization was at posterior wall $(70 \%$ of the cases) [24]. Given these findings we would expect that the AP2 view would perform well for detection of abnormalities for the aforementioned studies, in agreement with our study, though the performance characteristics may differ by cohort composition.

An important limitation of this study is that we relied on the use of structured echo reports for the designation of LV dysfunction and RWMA. We did not review any images to verify the accuracy of LVEF or RWMA reported and acknowledge that intra and interobserver variability in reporting of RWMA can be significant [25-27] in addition to the potential for mislabeling. Reported values for interobserver agreement for reporting of LV segment scores varies from 76 to $100 \%$ with intraobserver agreement of $82-92 \%$ in small studies [26, 27]. Furthermore, the issue of clinical disagreement is likely accentuated in settings of poor image quality, something that was not evaluated in the current study. That being said, these were clinical reports that were used in patient care, interpreted by advanced trained echocardiographers, scanned by professional sonographers and would generally be regarded as the reference standard. Although these findings may be most relevant to settings of limited cardiac ultrasound performed by clinicians, we recognize that there will be differences in the scanning and diagnostic ability of the broad range of physicians who perform limited cardiac ultrasound that is not captured by this study. The abnormality that we focused on was LV systolic dysfunction and therefore these results do not apply to the detection of other important pathology such as right heart dysfunction, valve disease, or pericardial effusion.

\section{Conclusions}

We demonstrate that in TTE studies with documented LV dysfunction, single-views will contain at least 1 abnormal LV segment $82-93 \%$ of the time. We also show that there is a slight variation in terms of LV wall motion abnormality by sex which may represent difference in distribution of coronary artery disease between males and females. Future 
studies examining image quality, ease of view acquisition, and sensitivity for detection of other abnormalities including pericardial effusion, right ventricular dysfunction, and major valve disease would be of value to determine the optimal views to learn and acquire in limited echo and cardiac POCUS.

Supplementary Information The online version contains supplementary material available at https://doi.org/10.1007/s10554-021-02460-4.

Funding The corresponding author receives research salary support from the Vancouver Coastal Health Research Institute Centre for Heart and Lung Health.

Data availability Data is not publicly available.

Code availability Not applicable.

\section{Declarations}

Conflict of interest The authors declare that they have no conflict of interest to disclose.

\section{References}

1. Johri AM, Durbin J, Newbigging J, Tanzola R, Chow R, De S et al (2018) Cardiac point-of-care ultrasound: state of the art in medical school education. J Am Soc Echocardiogr. https://doi.org/10. 1016/j.echo.2018.01.014

2. Haskins SC, Bronshteyn Y, Perlas A, El-Boghdadly K, Zimmerman J, Silva M et al (2021) American Society of Regional Anesthesia and Pain Medicine expert panel recommendations on point-of-care ultrasound education and training for regional anesthesiologists and pain physicians-part I: clinical indications. Reg Anesth Pain Med. https://doi.org/10.1136/rapm-2021-102560

3. Lambrecht JE, Zhang K, Tierney DM, Millner P, Giovannini $\mathrm{D}$, Barron $\mathrm{K}$ et al (2021) Integration of point of care ultrasound education into the internal medicine core clerkship experience. J Ultrasound Med. https://doi.org/10.1002/jum.15702

4. Olszynski P, Kim D, Chenkin J, Rang L (2018) The core emergency ultrasound curriculum project: a report from the Curriculum Working Group of the CAEP Emergency Ultrasound Committee. CJEM 20(2):176-182. https://doi.org/10.1017/cem.2017. 44

5. Lopresti CM, Jensen TP, Dversdal RK, Astiz DJ (2019) Point-ofcare ultrasound for internal medicine residency training: a position statement from the alliance of academic internal medicine. Am J Med 132(11):1356-1360. https://doi.org/10.1016/j.amjmed.2019. 07.019

6. Arntfield R, Millington S, Ainsworth C, Arora R, Boyd J, Finlayson $\mathrm{G}$ et al (2014) Canadian recommendations for critical care ultrasound training and competency. Can Respir J 21(6):341-345

7. Hanson MG, Chan B (2021) The role of point-of-care ultrasound in the diagnosis of pericardial effusion: a single academic center retrospective study. Ultrasound J. https://doi.org/10.1186/ s13089-021-00205-x

8. Sheth A, Dabo-Trubelja A (2021) Perioperative focused cardiac ultrasound: a brief report. J Anesth Crit Care 13(1):55-60

9. Fitzgibbon JB, Lovallo E, Escajeda J, Radomski MA, Martin-Gill C (2019) Feasibility of out-of-hospital cardiac arrest ultrasound by EMS physicians. Prehosp Emerg Care 23(3):297-303. https:// doi.org/10.1080/10903127.2018.1518505

10. Moulson N, Jaff Z, Wiltshire V, Taylor T, O'Connor HM, Hopman WM et al (2019) Feasibility and reliability of nonexpert POCUS for cardiovascular preparticipation screening of varsity athletes: the SHARP protocol. Can J Cardiol 35(1):35-41. https://doi.org/ 10.1016/j.cjca.2018.11.003

11. Bøtker MT, Jacobsen L, Rudolph SS, Knudsen L (2018) The role of point of care ultrasound in prehospital critical care: a systematic review. Scand J Trauma Resusc Emerg Med. https:// doi.org/10.1186/s13049-018-0518-x

12. Razi R, Estrada JR, Doll J, Spencer KT (2011) Bedside handcarried ultrasound by internal medicine residents versus traditional clinical assessment for the identification of systolic dysfunction in patients admitted with decompensated heart failure. J Am Soc Echocardiogr 24(12):1319-1324. https://doi.org/10. 1016/j.echo.2011.07.013

13. Cullen MW, Blauwet LA, Vatury OM, Mulvagh SL, Behrenbeck TR, Scott CG et al (2014) Diagnostic capability of comprehensive handheld vs transthoracic echocardiography. Mayo Clin Proc 89(6):790-798. https://doi.org/10.1016/j.mayocp.2013.12. 016

14. Johnson BK, Tierney DM, Rosborough TK, Harris KM, Newell MC (2016) Internal medicine point-of-care ultrasound assessment of left ventricular function correlates with formal echocardiography. J Clin Ultrasound 44(2):92-99. https://doi.org/10.1002/jcu. 22272

15. Johri AM, Galen B, Kirkpatrick JN, Lanspa M, Mulvagh S, Thamman R (2020) ASE statement on point-of-care ultrasound during the 2019 novel coronavirus pandemic. J Am Soc Echocardiogr 33(6):670-673. https://doi.org/10.1016/j.echo.2020.04.017

16. Hussain A, Via G, Melniker L, Goffi A, Tavazzi G, Neri L et al (2020) Multi-organ point-of-care ultrasound for COVID-19 (PoCUS4COVID): international expert consensus. Crit Care. https://doi.org/10.1186/s13054-020-03369-5

17. Lewis D, Rang L, Kim D, Robichaud L, Kwan C, Pham C et al (2019) Recommendations for the use of point-of-care ultrasound (POCUS) by emergency physicians in Canada. CJEM 21(6):721726. https://doi.org/10.1017/cem.2019.392

18. Kirkpatrick JN, Grimm R, Johri AM, Kimura BJ, Kort S, Labovitz AJ et al (2020) Recommendations for Echocardiography Laboratories Participating in Cardiac Point of Care Cardiac Ultrasound (POCUS) and Critical Care Echocardiography Training: report from the American Society of Echocardiography. J Am Soc Echocardiogr 33(4):409-22.e4. https://doi.org/10.1016/j.echo. 2020.01.008

19. Kendall JL, Bahner DP, Blaivas M, Budhram G, Dean AJ, Fox JC (2016) Emergency ultrasound imaging criteria compendium. Ann Emerg Med 68(1):e11-e48. https://doi.org/10.1016/j.annem ergmed.2016.04.028

20. Cholley BP, Mayo PH, Poelaert J (2011) International expert statement on training standards for critical care ultrasonography. Intensive Care Med 37(7):1077-1083. https://doi.org/10.1007/ s00134-011-2246-9

21. Levitov A, Frankel HL, Blaivas M, Kirkpatrick AW, Su E, Evans D et al (2016) Guidelines for the appropriate use of bedside general and cardiac ultrasonography in the evaluation of critically ill patients-part ii: cardiac ultrasonography. Crit Care Med 44(6):1206-1227. https://doi.org/10.1097/ccm.0000000000 001847

22. Lang RM, Badano LP, Mor-Avi V, Afilalo J, Armstrong A, Ernande L et al (2015) Recommendations for cardiac chamber quantification by echocardiography in adults: an update from the American Society of Echocardiography and the European Association of Cardiovascular Imaging. J Am Soc Echocardiogr 28(1):1-39.e14. https://doi.org/10.1016/j.echo.2014.10.003 
23. Davis MB, Shafton A, Desai A, Childers D, Bach DS (2014) Reliable exclusion of acute coronary syndrome among hospitalized patients with elevated troponin. Clin Cardiol 37(7):395-401. https://doi.org/10.1002/clc.22263

24. Bergmann I, Büttner B, Teut E, Jacobshagen C, Hinz J, Quintel $M$ et al (2018) Pre-hospital transthoracic echocardiography for early identification of non-ST-elevation myocardial infarction in patients with acute coronary syndrome. Crit Care. https://doi.org/ 10.1186/s13054-017-1929-1

25. Hoffmann R, Von Bardeleben S, Kasprzak JD, Borges AC, Ten Cate F, Firschke C et al (2006) Analysis of regional left ventricular function by cineventriculography, cardiac magnetic resonance imaging, and unenhanced and contrast-enhanced echocardiography. J Am Coll Cardiol 47(1):121-128. https://doi.org/10.1016/j. jacc.2005.10.012
26. Galema TW, Van De Ven ART, Soliman OII, Van Domburg RT, Vletter WB, Van Dalen BM et al (2011) Contrast echocardiography improves interobserver agreement for wall motion score index and correlation with ejection fraction. Echocardiography 28(5):575-581. https://doi.org/10.1111/j.1540-8175.2010.01379.x

27. Bjørnstad K, Al Amri M, Lingamanaicker J, Oqaili I, Hatle L (1996) Interobserver and intraobserver variation for analysis of left ventricular wall motion at baseline and during low- and high-dose dobutamine stress echocardiography in patients with high prevalence of wall motion abnormalities at rest. J Am Soc Echocardiogr 9(3):320-328. https://doi.org/10.1016/s08947317(96)90147-9

Publisher's Note Springer Nature remains neutral with regard to jurisdictional claims in published maps and institutional affiliations. 